\title{
Management of aorto-esophageal fistula secondary after thoracic endovascular aortic repair: a review of literature
}

\author{
Kaname Uno $^{1,2} \cdot$ Tomoyuki Koike $^{1} \cdot$ Seiichi Takahashi $^{2} \cdot$ Daisuke Komazawa $^{2}$ • \\ Tooru Shimosegawa ${ }^{1}$
}

Received: 28 May 2017/ Accepted: 6 July 2017/Published online: 1 August 2017

(C) Japanese Society of Gastroenterology 2017

\begin{abstract}
Aorto-esophageal fistula (AEF) is a rare and lethal entity, and the difficulty of making diagnosis of AEF is well-known. As promising results in the short-term effectiveness of thoracic endovascular aortic repair (TEVAR) promote its usage, the occurrence of AEF after TEVAR (post-TEVAR AEF) increases as one of the major complications. Therefore, we provide a review concerning the management strategy of post-TEVAR AEF. Although its representative symptom was reported as the triad of mid-thoracic pain and sentinel hematemesis followed by massive hematemesis, the symptom-free interval between sentinel hemorrhage and massive exsanguination is unpredictable. However, the physiological condition represents a surgical contraindication. Accordingly, early diagnosis is important, but either CT or esophago-gastroduodenoscopy rarely depicts a typical image. The formation of post-TEVAR AEF might be associated with the infection of micro-organisms, which is uncontrollable with anti-biotic administration. The current first-line strategy is combination therapy as follows, (1) to control bleeding by TEVAR in the urgent phase, and (2) radical debridement and aortic/esophageal re-construction in the semi-urgent phase. In view of the high mortality and morbidity rate, it is proposed that the choice in treatment strategies might be affected by patient's condition, size of the wall defects and the etiology of AEF. Practically, we should keep in mind
\end{abstract}

Kaname Uno

kaname.uno.c3@tohoku.ac.jp

1 Division of Gastroenterology, Tohoku University Hospital, 11 Seiryo-cho, Aoba-ku, Sendai, Miyagi 981-8574, Japan

2 Department of Gastrointestinal and Community Medicine, Tohoku University, 16 Kuzehara Uchigo-mimaya cho, Iwaki, Fukushima 973-8555, Japan the importance of making an early diagnosis and, once a suspicious symptom has occurred in a patient with a history of TEVAR, the existence of post-TEVAR AEF should be suspected. A prospective registry together with more developed technologies will be needed to establish a future strategy.

Keywords Aorto-esophageal fistula - Thoracic endovascular aortic repair - Thoracic aortic aneurysm . Endoscopy $\cdot$ Surgery

$\begin{array}{ll}\begin{array}{l}\text { Abbreviations } \\ \text { AEF }\end{array} & \text { Aorto-esophageal fistula } \\ \text { TEVAR } & \text { Thoracic endovascular aortic repair } \\ \text { post-TEVAR AEF } & \text { AEF after TEVAR } \\ \text { TAA } & \text { Thoracic aortic aneurysm } \\ \text { CT } & \text { Computed tomography } \\ \text { EGD } & \text { Esophago-gastro-duodenoscopy } \\ \text { HR-CT } & \text { High-resolution CT } \\ \text { CTA } & \text { CT angiography } \\ \text { UGIS } & \text { Upper gastrointestinal series } \\ \text { CPB } & \text { Cardio-pulmonary bypass } \\ \text { PEG } & \text { Percutaneous gastrostomy } \\ \text { SEMS } & \text { Self-expanding metallic stents } \\ \text { OTSC } & \text { Over-the-scope clip }\end{array}$

\section{Introduction}

Aorto-esophageal fistula (AEF) is a rare entity representing about $10 \%$ of aorto-enteric fistulas [1], but it is a lifethreatening condition that can cause massive bleeding and sepsis. A previous study estimated that $60 \%$ of patients suffering from AEF might die within 6 months after the onset of their symptoms [2]. In addition, the difficulty of 
making a pre-operative diagnosis of AEF has been emphasized [3], as Carter et al. demonstrated in the past that, in all 24 of their patients, a definitive diagnosis of its presence could be made at the post-mortem [4]. Here, we provide a review of the diagnostic and therapeutic strategies for AEF after thoracic endovascular aortic repair (TEVAR, post-TEVAR AEF), in order to increase awareness and improve the outcome.

\section{Etiologies of aorto-esophageal fistula}

Previously, Hollander and Quick reviewed 500 AEF cases in 1991 and demonstrated that untreated thoracic aortic aneurysm (TAA; $54.2 \%$ ), foreign body ingestion (19.2\%) and invasion of malignant esophageal tumor (17\%) were three major causes of $\mathrm{AEF}$, despite the fact that prosthetic graft-associated complications were present in only a small proportion of such cases (1.4\%). Further studies described other causes including esophageal reflux, tuberculosis, congenital abnormalities and traumatic injuries [2, 5-7]. Among traumatic injuries, the numbers of iatrogenic complications, such as failure of the sutures for esophagectomy, esophageal stent placement, nasogastric tube insertion, endoscopic esophageal biopsies and (chemo) radiation therapy for lung/esophageal cancer, have been increasing [2, 8-13]. Accordingly, the etiology of AEF has changed together with gradual improvements in diagnostic/treatment technologies.

From the point of view of the etiology of AEF, there are two groups. While secondary AEF is defined as AEF that occurs after aortic reconstructive surgery with prosthetic grafts, others are assumed to represent the primary AEF. The incidence of the former was reported to be as high as $1.6 \%$, while those of the latter were much rarer $[14,15]$.

As for the secondary AEF, the recent increase in the occurrence of post-TEVAR AEF is striking. In the past, TEVAR was positioned as the main treatment strategy for TAA or traumatic aortic transection after its clinical introduction in 1994 [16]. Various studies demonstrated that the minimally invasive procedure of TEVAR might allow rapid control of blood loss even in patients with hemodynamic instability $[17,18]$, and that the perioperative morbidity of TEVAR during an urgent condition was significantly better than that of emergency open surgery [19]. After the usefulness of TEVAR in closing a fistula between the aorta and an adjacent organ was reported in 1996 [20], numerous promising results in its short-term effectiveness have encouraged its clinical use, and the indications of TEVAR have expanded to AEF.

However, the long-term results of TEVAR remain unknown. Several complications of TEVAR, including paraplegia, stroke, post-implantation embolisms and AEF, have been reported [21-30]. The incidence of post-TEVAR AEF was reported to be $1.7-1.9 \%$, comparable to the incidence after open surgery for TAA, resulting in a high rate of re-stenting for AEF recurrence [21-23]. As the clinical use of TEVAR has been widespread, a recent metaanalysis study demonstrated that primary malignant/benign esophageal disease, a prior treatment of open surgery or TEVAR of TAA, untreated TAA or a foreign body might be associated with the AEF formation in 23.6, 20.8, 20.8 or $12.5 \%$ of 72 AEF patients with the initial treatment of TEVAR, respectively [28]. Therefore, in the near future, the incidence of post-TEVAR AEF as one of the major complications of TEVAR may increase further by the widespread use of TEVAR for TAA, AEF and postTEVAR AEF.

\section{Symptom of post-TEVAR AEF}

Chiari's triad of mid-thoracic pain and sentinel hematemesis followed by massive hematemesis was reported as the representative symptom of AEF [31-36]. In an original study of 500 AEF cases, Hollander and Quick mentioned that hematemesis, chest pain or dysphagia were observed in 63,58 and $42 \%$ of the subjects, respectively [2]. Steffes and O'Leary reported that massive fatal hemorrhage within 1 week after the onset of sentinel bleeding was observed in about $80 \%$ of AEF cases [37]. Amin et al. suggested that the spontaneous cessation of sentinel hemorrhage may be caused by temporary occlusion of the fistula due to spasm of the arterial wall, intra-vascular hypotension from the initial hemorrhage, or occlusion of the fistula by a peri-aortic hematoma, which may be digested later by infectious agents or digestive fluid [36].

Unfortunately, the occurrence of massive hemorrhage due to post-TEVAR AEF is not predictable. While most cases seem to occur within 1-16 months after the TEVAR procedure, Kouritas et al. reported one case of AEF that developed 6 years after TEVAR [38]. Other less frequent presentations of post-TEVAR AEF were dysphagia due to extra-luminal compression of the esophageal wall or highgrade fever due to mediastinitis, infection of the surgical/ endovascular graft or sepsis [22, 34, 35]. A recent review revealed that hematemesis, hypovolemic shock or systemic infection were observed in $86.1,60.9$ or $21.7 \%$ of 72 patients with post-TEVAR AEFs, respectively [28].

\section{Mechanism of post-TEVAR AEF}

There are some hypotheses about the pathological mechanism of post-TEVAR AEF, although it remains unknown. Previous studies suggested that the radical force of a rigid 
graft expansion might cause direct erosion through the aorta into the esophagus, or pressure necrosis in the native aortic wall. As a result, the inflammation in the hematoma, mechanical compression by a large aneurysm or the force from the graft expansion might cause higher pressure in the mediastinum, resulting in further expansion of pressure necrosis due to occlusion of the aortic side branches that feed the esophagus [31, 39, 40]. Additionally, the largest risk factor for the development of its formation was reported to be infection of the implanted graft. Actually, the incidence of infection around the graft expanding to the esophagus was reported to be $0.5-5 \%$, and has been proposed as having a central role in the development of postTEVAR AEF [22]. In a review article, micro-organisms were reported in $43.2 \%$ of the patients after TEVAR for $\mathrm{AEF}$, and, in $31.2 \%$ of the patients with inflammation, highly virulent pathogens, such as methicillin-resistant Staphylococcus aureus, Streptococcus spp., Gram-negative species, such as Pseudomonas and Klebsiella, or Coxiella burnetti were isolated $[28,41]$. These findings suggest that micro-organisms associated with the post-TEVAR AEF formation could hardly be controlled with general use of antibiotic therapy [32].

\section{Importance of early diagnosis of post-TEVAR AEF}

Once sentinel hematemesis has occurred in any patient with a history of thoracic surgery or TEVAR, they should be highly suspected for the possibility of post-TEVAR $\mathrm{AEF}$ in the clinical setting.

As for the basic screening tools, laboratory tests for detecting systemic inflammation, anemia as well as blood cultures should be employed, and chest x-ray can demonstrate a widened mediastinum, tracheal deviation with a tortuous aorta, and/or calcification [42].

Many studies proposed that both computed tomography (CT) and esophago-gastro-duodenoscopy (EGD) might be powerful modalities for its diagnosis. Considering that AEF is often located on the physiologically narrowing parts due to compression of the aortic arch, the location of abnormal findings in the images may provide important cues of its existence.

As for the CT findings (Fig. 1), it rarely depicts the fistula itself, but it clearly shows some suggestive signs, such as the presence of air bubbles within the thrombus in the aorta or around the thickened esophageal/bronchial wall, persistent/expanding fluid collection around the graft, irregular density components of soft tissue more than 6 months after the TEVAR implantation, loss of the aortoesophageal fat plane, or the extravasation of contrast outside the aortic lumen [10, 43]. High-resolution CT (HR-
CT) provides a higher accuracy rate for AEF with its clear view of the mediastinum components, and, further, it can help to assess the condition of a pseudo-aneurysm or mediastinitis together with the extent of soft tissue involvement, so as to enable planning for aspiration or drainage of the inflamed components [44]. CT angiography (CTA) can also directly depict extravasation of the contrast material outside the aorta [31, 45-47]. Accordingly, CT is usually used for the initial investigation in cases suspicious for AEF, though such typical findings may be rarely seen.

On the other hand, it has been suggested that EGD might be the most sensitive and specific modality for making a real-time diagnosis (Fig. 2) [48]. The most common EGD finding of $\mathrm{AEF}$ is a sub-mucosal tumor-like protrusion with/without clots/debris due to extrinsic compression on the esophageal wall. Other reported EGD findings included ulcerative lesions, oozing from a pin-hole erosion or a pulsating protrusion with fistula, which might be related to graft exposure $[35,49,50]$. Interestingly, AEF is usually observed between the posterior to left lateral wall of the upper/middle-esophagus in the endoscopic findings. Sosnowik et al. proposed that flexible endoscopy is a valuable diagnostic modality not only from the point of view that the awareness of suggestive signs in the upper/middle of the esophagus might promote timely re-examination [51], but also because it might exclude other possible causes of upper gastrointestinal bleeding. However, some investigators pointed out its limitations in terms of its low sensitivity and risk of complications during the examination, such as rupture of the TAA as well as worsening of the co-existing cardio-pulmonary disease. Miller et al. reported that the sensitivity of even conventional endoscopy was only $38 \%$ [52]. Furthermore, Chiba et al. suggested that the intubation of a trans-nasal small caliber endoscopy might be safer than a trans-oral conventional endoscopy for AEF patients in poor condition in an urgent situation, but the sensitivity of the former is much lower than that of the latter [35, 53].

As another luminal examination, an upper gastrointestinal series (UGIS) is also generally used to make an easy diagnosis of AEF, when it visualizes extravasation of the contrast through the fistula or luminal irregularities due to hematoma or external compression [54]. However, its sensitivity is much lower than those of CT or EGD.

Accordingly, we should be mindful of the limitations of these modalities to demonstrate the presence of AEF. There was a case report in which the diagnosis was confirmed retrospectively after the second session of the TEVAR procedure, despite the fact that the initial examination of UGIS and CTA missed the presence of post-TEVAR AEF due to the lack of typical findings [47]. Therefore, for early diagnosis prior to the occurrence of lethal complications, it is important to keep in mind that once suspicious symptoms have occurred in any patient with a history of TEVAR, we should not rule out the 
Fig. 1 a An enhanced CT on admission showed a soft tissue density mass with air bubbles around the graft (arrow). b On post-admission day 5 , the laboratory data had improved by anti-biotic administration, but the re-examined CT presented a high-density spot (arrow) in contact with the esophagus around the graft
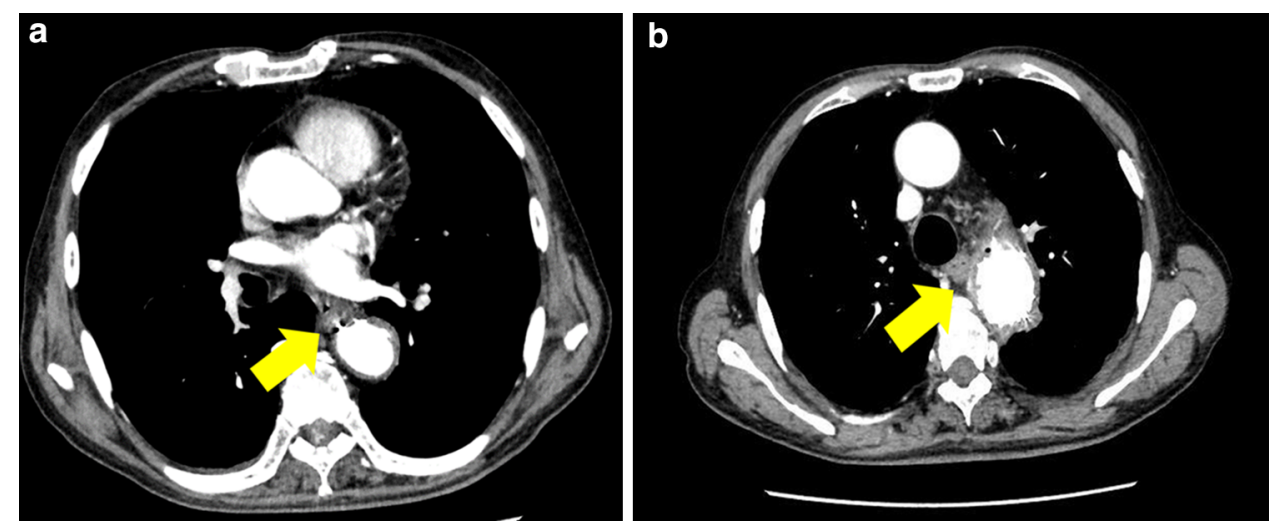

Fig. 2 EGD revealed a white pus discharge from a small ulcerative lesion without any signs of acute bleeding in the mid-esophagus, and the background mucosa was longsegment Barrett's esophagus (C8M10) with no suspicion of dysplastic Barrett's esophagus
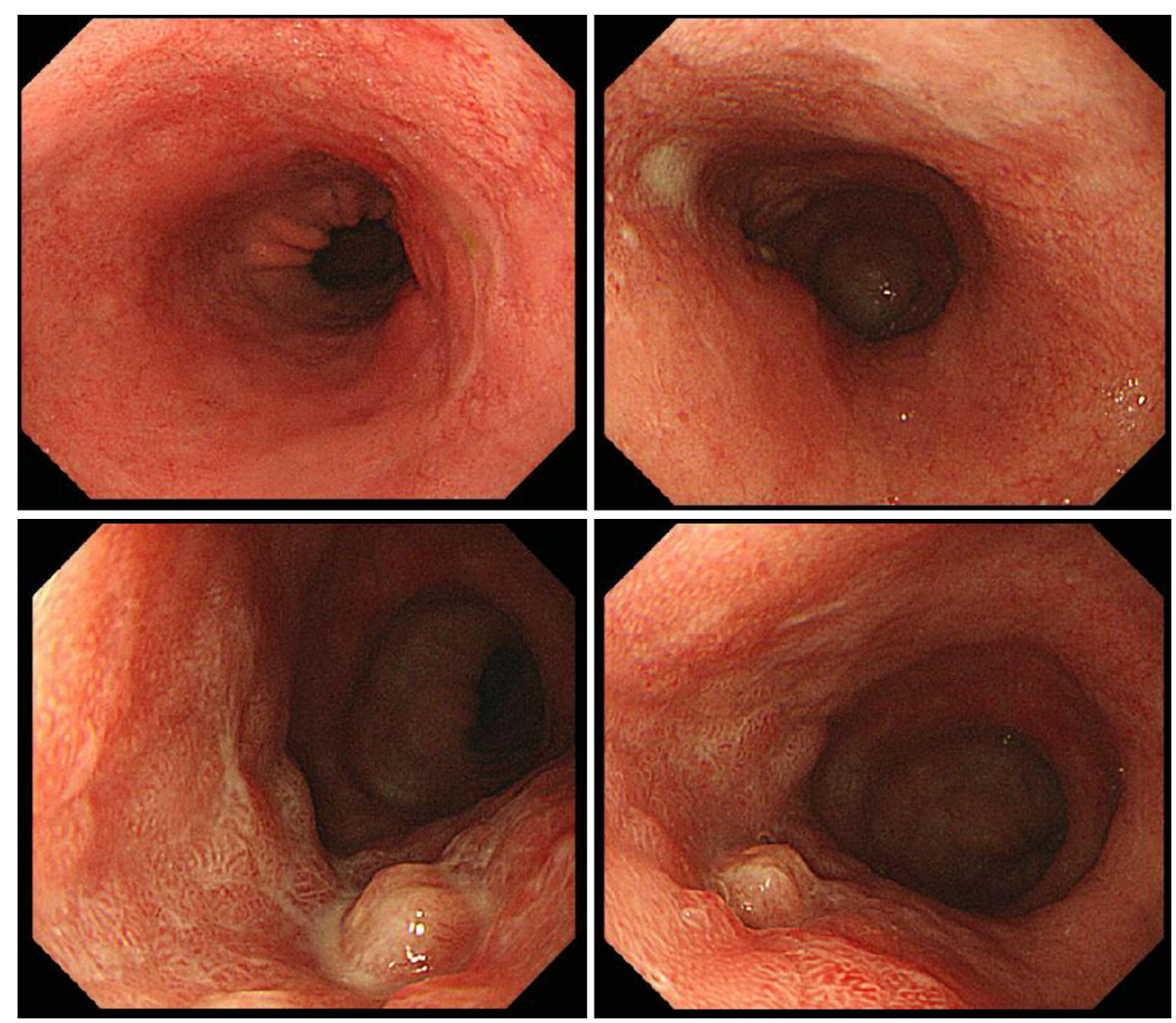

possibilities of AEF, in spite of its rare occurrence as well as occult findings in several pre-operative examinations.

\section{Principle treatment strategy of post-TEVAR AEF}

Once an AEF has developed, it can rapidly cause lethal complications. Because of the poor condition of their basal physiology, in most AEF patients in an urgent condition, surgical intervention is contraindicated. Therefore, there remain many issues important for improving the effectiveness of treatment.

\section{Radical surgical approach}

A first case report in 1980 described that a patient with AEF caused by a foreign body was successfully treated by surgical treatment, which consisted of the cross-clamping of the descending TAA followed by primary closure of the fistula [55]. The current surgical approach, such as (i) primary resection of the esophagus, (ii) debridement of the contaminated tissues followed by abundant lavage of the mediastinum and (iii) in situ reconstruction of the aorta with a prosthesis, has been established as the first-line surgical strategy for AEF [25, 56-59]. 
With the development of new surgical techniques and devices, primary closure of small defects of the esophageal wall has become possible only in selected cases without gross contamination in the mediastinum. However, primary repair is reported to be frequently unsuccessful, due to the weakness of the wound and refractory defects of AEF, and, as a result, the breakdown from suturing defects might induce the recurrence of AEF [56]. Instead of wall repair, esophageal resection might eliminate the risk from disruption of the sutured fistula and minimize the recurrence of contamination through the fistula (Fig. 3). Therefore, most recent studies emphasized the importance of sub-total esophagectomy and ligation of the distal esophagus with a cervical esophagostomy and concomitant gastrostomy or jejunostomy to enable enteral access to bypass the fistula $[25,56]$. However, such management has a significant mortality of from 25 to $78 \%$ due to its massive invasiveness in patients in a physiologically poor condition [21, 34, 60-62]. Actually, the high rate of mortality and morbidity of this surgical approach might be associated with the nature of emergency surgery to access the damaged aorta in a contaminated field, as well as the need for an invasive procedure, such as thoracic aortic crossclamping or cardio-pulmonary bypass (CPB). In a recent study of 47 AEF patients, the newly established surgical strategies-esophagectomy, in situ reconstruction of the aorta, and additional wrapping of the fistula with a flapwere reported to be effective in the improvement of the mid-term mortality rate after the surgery [26]. Further development of surgical strategies will be needed.

\section{Combination therapy}

At present, the fundamental strategies involve combination therapy, as follows: (1) controlling fatal bleeding by TEVAR in the urgent phase, and (2) radical debridement of the contaminated area as well as aortic/esophageal reconstruction in the semi-urgent phase. In fact, previous studies demonstrated that emergency surgery or conventional therapy alone after TEVAR might be fatal.

\section{Controlling fatal bleeding from AEF}

AEF frequently causes sudden life-threatening hematemesis with circulatory collapse. The aim of the initial treatment of AEF in the urgent phase is to maintain hemodynamic stability in the patients who frequently suffer from co-existent cardiopulmonary disease. In the past, the insertion and inflation of a Sengstaken-Blakemore tube, or embolization with isobutyl-2-cyanoacrylate, was used to stop bleeding temporarily prior to surgery [63-66]. With recent developments in endovascular stenting techniques, TEVAR has become widespread for the initial hemostasis of AEF with favorable short-term outcomes. TEVAR facilitates hemodynamic stabilization in the urgent phase of AEF, resulting in reduction of the morbidity and mortality of radical surgery during the semi-urgent phase. The outcomes of TEVAR for AEF were reported to have a high success rate of $87.3 \%$ and a favorable 30-day mortality rate of $19.7 \%$ [28, 67]. Further, Kubota et al. recommended that TEVAR should be performed at the initial step in all patients with AEF, regardless of their hemodynamic stability on admission [25]. Actually, the symptomfree interval between sentinel hemorrhage and the following massive exsanguination is unpredictable. Accordingly, TEVAR is more useful than emergency open surgery for high-risk AEF patients in an urgent situation, but there is no available data about its durability for AEF patients.

In such AEF cases, the graft is usually positioned in the contaminated area during emergency treatment, although the material is not immune to infection. There is no consensus regarding the optimal aortic substitute, including the implantation of a Dacron graft, a PTFE graft and so on [68]. In other words, TEVAR itself does nothing to control the infectious condition or to heal the esophageal wall defect, leaving the patients at risk of graft infection and/or fistula recurrence and persistent mediastinitis. When the graft replacement is performed repeatedly in such a contaminated field, the recurrence of an endo-leak within the aneurysmal sac or the persisting graft infection may become refractory [30]. A previous meta-analysis study described that the recurrence of AEF and graft infection after TEVAR occurred in 13.8 and $15.2 \%$ of the patients, respectively [28], and that prolonged ineffective administration of intra-venous anti-biotic medicine was the most significant factor associated with a lower mortality in the multi-variate analyses, despite the lack of definitive strategies for post-operative anti-biotic usage. Previous studies pointed out that the outcome of conservative management alone after TEVAR might be fatal due to recurrent hemorrhage or sepsis [26, 28, 40, 68-72]. Accordingly, TEVAR should be performed only as a bridging treatment before radical surgery is planned to treat the inflamed lesions of AEF patients [69].

\section{Perioperative management prior to radical debridement}

After the primary TEVAR, intensive medical support, such as treatment for anemia, controlling infection by long-term administration of broad-spectrum antibiotics, medical blockade of gastric acid with proton-pump inhibitor, and nutrition by total enteral feeding via percutaneous gastrostomy (PEG)/jejunostomy, are required to improve the general condition. It is widely believed that once the physical condition has thereby improved, the patient should be referred to undergo radical surgery as soon as possible. 
Fig. 3 Post-operative specimens of the esophagus with AEF: the specimen obtained by esophagectomy (a), and the histological examination $(\mathbf{b}: \times 40, \mathbf{c}, \mathbf{d}: \times 100)$ showed fistula formation with abundant inflammatory cell infiltration in non-malignant metaplastic Barrett's epithelium
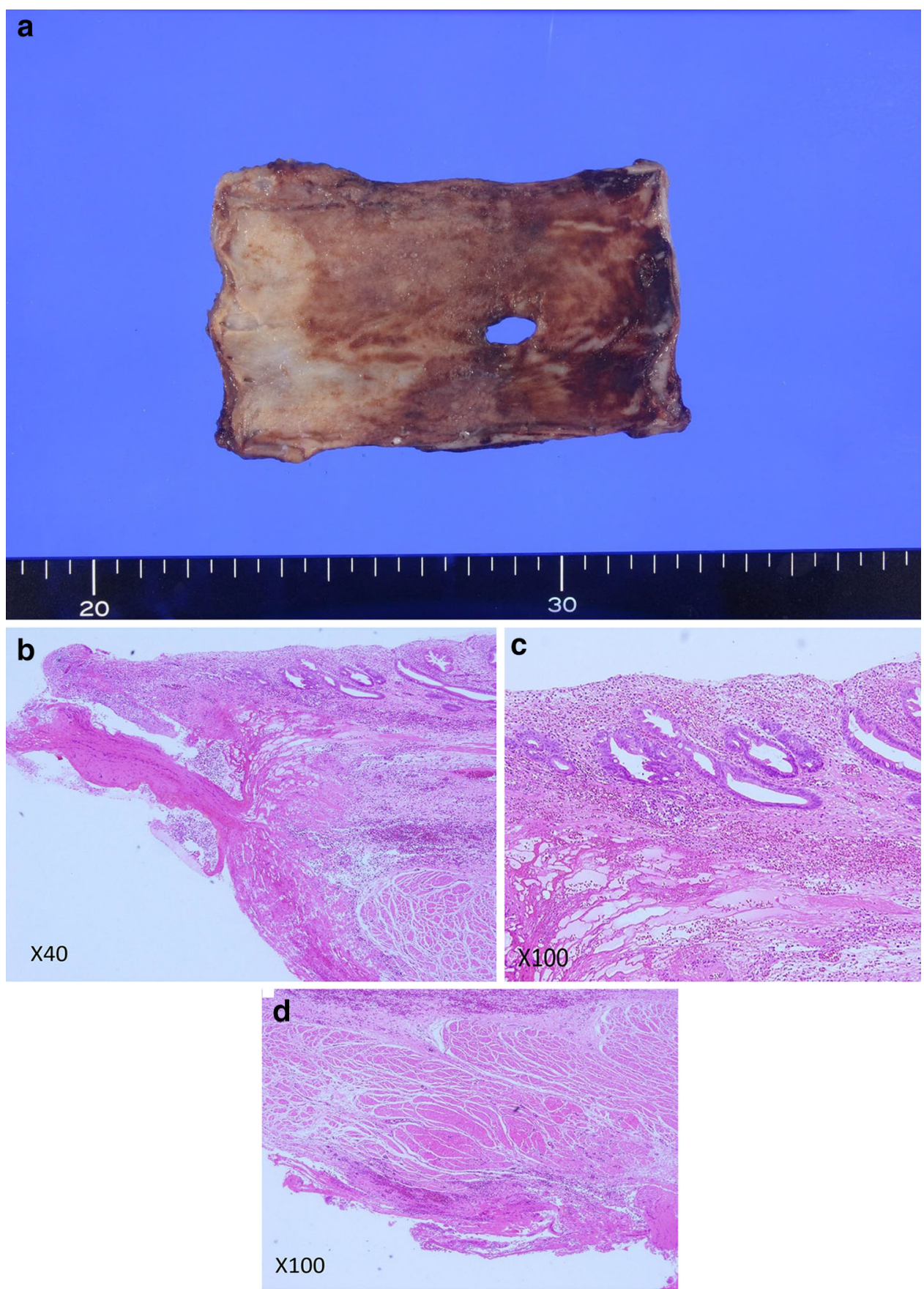

Despite the lack of consensus about the appropriate interval from TEVAR to radical surgery, Okita et al. proposed that the optimal timing was within 1 week; otherwise, the graft infection might progress [68].

\section{Surgical strategy after TEVAR}

Recent studies have emphasized that the combination therapy should be mandatory to cure post-TEVAR AEF $[68,69,73]$. A multi-center study of patients with postTEVAR AEF demonstrated no survivors at 1 year after
TEVAR alone [73]. Another study revealed that extensive debridement with simultaneous resection of the esophagus and in situ reconstruction of the aorta using CPB provided better outcomes than TEVAR alone in terms of no recurrence [68].

However, there is no established surgical method. A Japanese study of 16 patients with AEF (11 patients with TAA, 5 with esophageal cancer and 1 with fish bone penetration) described that after the initial treatment $(8$ patients treated by TEVAR, 2 by a bridge TEVAR to surgery, 2 by extra-anatomical bypass and 5 by in situ 
aortic reconstruction), there were 8 cases treated by esophagectomy, 7 cases by repair with an omental flap and 4 cases by simultaneous resection of the esophagus and in situ aortic reconstruction reinforced with a flap, and that there was a significant difference in the mortality rate not among the various surgical strategies, but among the AEF etiologies [68]. Additionally, in a meta-analysis, Canaud et al. described that only $11.1 \%$ of the 72 patients treated by TEVAR for AEFs were according to the scheduled strategy, and, finally, a total of $44.4 \%$ of the patients were treated by 1 or 2 -staged surgical procedures, resulting in 11 patients with post-operative death due to graft infection and 10 patients from AEF recurrence. They suggested that poor control of the inflamed lesions even with prolonged antibiotic treatment and/or incomplete surgical management might have caused the significantly higher rates of mortality and morbidity (Fig. 4) [28, 74].

\section{Palliative treatment for AEF}

Although TEVAR is usually performed as a bridging treatment, its successful use as a definitive treatment for limited cases has been reported $[73,75]$. Some patients whose conditions made them unlikely to tolerate surgical intervention were reported to survive with conservative treatment, consisting of anti-biotic therapy and percutaneous drainage/ aspiration with/without TEVAR, during a limited period, when they had only a small fistula with a small amount of fluid collection around the fistula, and with no evidence of sepsis [31, 42]. Kasai et al. reported a case of post-TEVAR AEF with advanced hepatocellular carcinoma that survived after conservative management alone without TEVAR for 14 months after the initial bleeding event [76]. Numan et al. described a case of post-TEVAR AEF related to an infected aneurysm that was treated by CT-guided insertion of a

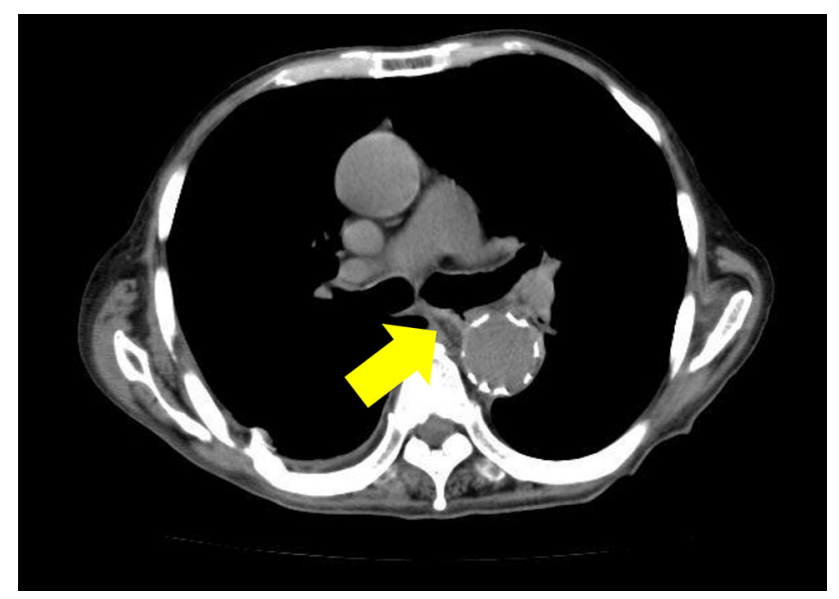

Fig. 4 Broncho-mediastinum fistula formation (arrow) after surgical intervention followed by conventional treatment with broad-spectrum antibiotics and enteral nutrition therapy drainage catheter [77]. Burks Jr. et al. suggested in their seven-case series that the combination therapy of TEVAR followed by long-term anti-biotic treatment and percutaneous drainage might be a safe method [42, 78].

Other groups demonstrated the usefulness of self-expanding metallic stents (SEMS) to cover the fistula from the esophageal side. Eggebrecht et al. reported that two of three patients with post-TEVAR AEF survived for 2-6 months after the insertion of SEMS [30]. Onodera et al. and Tao et al. reported that the implantation of covered SEMS followed by the life-long use of broadspectrum antibiotics prohibited re-bleeding and controlled inflammation in post-TEVAR AEF patients [24, 42]. However, stent migration due to incomplete sealing on the smooth surface of a non-tumorous esophagus may require repositioning or replacement of the stent. A previous study demonstrated that $17 \%$ of 187 esophageal stent insertions required repositioning or replacement [23]. But, the development of a new-generation SEMS with anchors, such as the Niti-S esophageal stent and Double Niti-S ${ }^{\mathrm{TM}}$ esophageal stent (Taewoong Medical, Seoul, South Korea), may be able to reduce risk of such migration. Moreover, considering that the over-the-scope clip (OTSC) system was developed for closing esophageal fistulas or for anchoring SEMS under direct view of endoscopic examination [80], it may be assumed that SEMS anchored with the OTSC system can be used as an alternative strategy for SEMS together with endoscopic ultrasound-fine needle aspiration techniques for drainage [81, 82].

Accordingly, TEVAR and/or esophageal stenting followed by anti-biotic treatment and/or percutaneous drainage could be an option for a palliative strategy for a substantial period [47]. Further developments of endoscopic technologies can be expected to contribute to the improving the outcome of palliative strategies.

\section{Summary of current treatment strategy}

After TEVAR is performed as an urgent treatment, complete eradication by debridement of all contaminated tissues followed by abundant lavage of the mediastinum is recommended as radical surgery. In contrast, conservative treatment alone after TEVAR is controversial, because endo-leakage after stent placement on the weakened wall of the aorta might promote the continuation of inflammatory changes. However, previous studies demonstrated that the rate of mortality after surgery for secondary AEF (78\%) was higher than that for primary AEF (61\%) [21]. Therefore, some investigators proposed that the appropriate choice in treatment strategies might be depend on the patient's condition, size of the wall defects and the etiology of AEF [47, 79]. 


\section{Perspectives}

Due to the low incidence of post-TEVAR AEF, previous studies presented relatively favorable outcomes for the current strategy during a limited period, despite their poor prognosis [60]. However, there is a lack of uniformity in the patients ' demographics, etiologies of AEF as well as treatment strategies, such as antibiotic usage, TEVAR device or surgical method. Furthermore, most of the data obtained from case reports and case series may include some bias. Therefore, it is hard for us to determine the most suitable management strategy for post-TEVAR AEF, the incidence of which will likely increase in the near future. Accordingly, a prospective registry with a large number of post-TEVAR AEF patients will help to determine the precise results of the combination treatment, and to establish an ideal strategy.

\section{Conclusions}

Although AEF is a fatal entity, the feasibility of ongoing strategies has not been fully elucidated because of its small incidence. As post-TEVAR AEF can be one of the major iatrogenic complications, early diagnosis prior to the onset of lethal symptoms is critically important. Although various diagnostic modalities have been used to make an early diagnosis, none of them seems to have enough sensitivity or specificity to achieve accuracy [45]. Therefore, to provide promising treatment for post-TEVAR AEF patients in the current setting, we should be aware of the possibility for any patient with a treatment history of TEVAR to require urgent TEVAR followed by radical surgery. Further developments in diagnostic imaging and treatment technologies for post-TEVAR AEF are needed.

Figure legends: Representative photos of a post-TEVAR AEF case in our institution.

A 77-year-old man presented with nausea and highgrade fever. He had a history of a TEVAR for thoracic aortic aneurysm at the age of 75 , and, with regular use of anti-thrombotic drugs, he underwent regular surveillance after TEVAR every 6 months. From the age of 76 , he took on-demand proton pump inhibitor for gastro-esophageal reflux disease. On admission, he was hemodynamically stable without any sign of active bleeding, and presented elevated laboratory markers of systemic inflammation with a high value of procalcitonin, despite negative results at several sessions of blood culture. With intra-venous administration of ceftriaxone followed by oral administration of cefcapene pivoxil hydrochloride, several examinations were performed during starvation to investigate the infectious focus. We concluded that his symptom was caused by mediastinitis due to post-TEVAR AEF, which might be induced by the rupture of an inflamed TAA. Although his condition was relatively poor, we performed esophagectomy with a cervical esophagostomy and concomitant gastrostomy and jejunostomy on post-admission day 19. Thereafter, it was not possible to perform the planned aortic/esophageal re-construction. Instead, his condition gradually got worse, resulting in sepsis due to the formation of broncho-mediastinum fistula.

\section{Compliance with ethical standards}

Conflict of interest Kaname Uno, Seiichi Takahashi, Daisuke Komazawa, Tomoyuki Koike and Tooru Shimosegawa declare that they have no conflicts of interest.

Human rights All procedures followed have been performed in accordance with the ethical standards laid down in the 1964 Declaration of Helsinki and its later amendments.

Informed consent Informed consent was obtained from the patients whose images were used as a representative case of aorto-esophageal fistula in the manuscript.

\section{References}

1. Sinar DR, DeMaria A, Kataria YP, et al. Aortic aneurysm eroding the esophagus. Case report and review. Am J Dig Dis. 1977;22:252-4.

2. Hollander JE, Quick G. Aortoesophageal fistula: a comprehensive review of the literature. Am J Med. 1991;91:279-87.

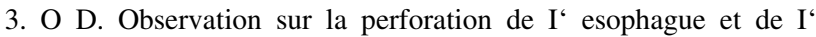
aortethroracique par une portion d'os oval: avec des reflexions. J Univ Sci Med 1818;9:357-363.

4. Carter R, Mulder GA, Snyder EN Jr, et al. Aortoesophageal fistula. Am J Surg. 1978;136:26-30.

5. Podbielski FJ, Rodriguez HE, Zhu RY, et al. Aortoesophageal fistula secondary to reflux esophagitis. Dig Surg. 2007;24:66-7.

6. Lee OJ, Kim SH. Aortoesophageal fistula associated with tuberculous mediastinitis, mimicking esophageal Dieulafoy's disease. J Korean Med Sci. 2002;17:266-9.

7. Angelini A, Dimopoulos K, Frescura C, et al. Fatal aortoesophageal fistula in two cases of tight vascular ring. Cardiol Young. 2002;12:172-6.

8. Ahn M, Shin BS, Park MH. Aortoesophageal fistula secondary to placement of an esophageal stent: emergent treatment with cyanoacrylate and endovascular stent graft. Ann Vasc Surg. 2010;24:555-e1.

9. Oe K, Araki T, Nakashima A, et al. Aortoesophageal fistula following nasogastric tube placement. Clin J Gastroenterol. 2009;2:284-6.

10. Wiggins T, Chaudry MA, Vasas P, et al. Aorto-oesophageal fistula: an unusual complication of oesophageal biopsies. BMJ Case Rep 2011;2011. doi:10.1136/bcr.01.2011.3770.

11. Lee RY, Flaherty L, Khushalani NI, et al. Aorto-esophageal fistula: a rare fatal case caused by esophageal malignancy. J Gastrointest Oncol. 2010;1:64-5.

12. Parikh MP, Sherid M, Panginikkod S, et al. Radiation therapyinduced aortoesophageal fistula: a case report and review of literature. Gastroenterol Rep (Oxf). 2016;4:165-7. 
13. Tsushima T, Mizusawa J, Sudo K, et al. Risk factors for esophageal fistula associated with chemoradiotherapy for locally advanced unresectable esophageal cancer: a supplementary analysis of JCOG0303. Medicine (Baltimore). 2016;95:e3699.

14. Perks FJ, Gillespie I, Patel D. Multidetector computed tomography imaging of aortoenteric fistula. J Comput Assist Tomogr. 2004;28:343-7.

15. Dossa CD, Pipinos II, Shepard AD, et al. Primary aortoenteric fistula: part I. Ann Vasc Surg. 1994;8:113-20.

16. Dake MD, Miller DC, Semba CP, et al. Transluminal placement of endovascular stent-grafts for the treatment of descending thoracic aortic aneurysms. N Engl J Med. 1994;331:1729-34.

17. Chiesa R, Melissano G, Marone EM, et al. Endovascular treatment of aortoesophageal and aortobronchial fistulae. J Vasc Surg. 2010;51:1195-202.

18. Walsh SR, Tang TY, Sadat U, et al. Endovascular stenting versus open surgery for thoracic aortic disease: systematic review and meta-analysis of perioperative results. J Vasc Surg. 2008;47:1094-8.

19. Fattori R, Lovato L, Buttazzi K, et al. Extension of dissection in stent-graft treatment of type B aortic dissection: lessons learned from endovascular experience. $\mathrm{J}$ Endovasc Ther. 2005;12:306-11.

20. Chuter TA, Ivancev K, Lindblad B, et al. Endovascular stent-graft exclusion of an aortobronchial fistula. J Vasc Interv Radiol. 1996;7:357-9.

21. Chiesa R, Melissano G, Marone EM, et al. Aorto-oesophageal and aortobronchial fistulae following thoracic endovascular aortic repair: a national survey. Eur $\mathrm{J}$ Vasc Endovasc Surg. 2010;39:273-9.

22. Eggebrecht H, Mehta RH, Dechene A, et al. Aortoesophageal fistula after thoracic aortic stent-graft placement: a rare but catastrophic complication of a novel emerging technique. JACC Cardiovasc Interv. 2009;2:570-6.

23. Lee SH, Song PS, Kim WS, et al. A case of stent graft infection coupled with aorto-esophageal fistula following thoracic endovascular aortic repair in a complex patient. Korean Circ J. 2012;42:366-8.

24. Onodera M, Inoue Y, Fujino Y, et al. A case of secondary aortoesophageal fistula inserted a covered self-expanding esophageal stent to control gastrointestinal bleeding. Case Rep Gastrointest Med. 2013;2013:857135.

25. Kubota S, Shiiya N, Shingu Y, et al. Surgical strategy for aortoesophageal fistula in the endovascular era. Gen Thorac Cardiovasc Surg. 2013;61:560-4.

26. Akashi H, Kawamoto S, Saiki Y, et al. Therapeutic strategy for treating aortoesophageal fistulas. Gen Thorac Cardiovasc Surg. 2014;62:573-80.

27. Marone EM, Coppi G, Kahlberg A, et al. Combined endovascular and surgical treatment of primary aortoesophageal fistula. Tex Heart Inst J. 2010;37:722-4.

28. Canaud L, Ozdemir BA, Bee WW, et al. Thoracic endovascular aortic repair in management of aortoesophageal fistulas. J Vasc Surg. 2014;59:248-54.

29. Wang GJ, Fairman RM. Endovascular repair of the thoracic aorta. Semin Intervent Radiol. 2009;26:17-24.

30. Eggebrecht H, Baumgart D, Radecke K, et al. Aortoesophageal fistula secondary to stent-graft repair of the thoracic aorta. J Endovasc Ther. 2004;11:161-7.

31. Xi EP, Zhu J, Zhu SB, et al. Secondary aortoesophageal fistula after thoracic aortic aneurysm endovascular repair: literature review and new insights regarding the hypothesized mechanisms. Int J Clin Exp Med. 2014;7:3244-52.

32. Fang YH, Zhou XL, Liu JC. Aortoesophageal fistula (AEF): fatal upper gastrointesinal haemorrhage. Turk J Gastroenterol. 2014;25(Suppl 1):321-3.
33. Genuis ED, Kim DJ. A case of secondary aortoesophageal fistula. J Emerg Med. 2015;49:e193-4.

34. Muradi A, Yamaguchi M, Kitagawa A, et al. Secondary aortoesophageal fi stula after thoracic endovascular aortic repair for a huge aneurysm. Diagn Interv Radiol. 2013;19:81-4.

35. Chiba D, Hanabata N, Araki Y, et al. Aortoesophageal fistula after thoracic endovascular aortic repair diagnosed and followed with endoscopy. Intern Med. 2013;52:451-5.

36. Amin S, Luketich J, Wald A. Aortoesophageal fistula: case report and review of the literature. Dig Dis Sci. 1998;43:1665-71.

37. Steffes BC, O'Leary JP. Primary aortoduodenal fistula: a case report and review of the literature. Am Surg. 1980;46:121-9.

38. Kouritas VK, Dedeilias P, Sotiriou K, et al. Delayed presentation of aortoesophageal fistula after endovascular repair. Asian Cardiovasc Thorac Ann. 2016;24:51-3.

39. Girdauskas E, Falk V, Kuntze T, et al. Secondary surgical procedures after endovascular stent grafting of the thoracic aorta: successful approaches to a challenging clinical problem. J Thorac Cardiovasc Surg. 2008;136:1289-94.

40. Morisaki A, Hirai H, Sasaki Y, et al. Aortoesophageal fistula after endovascular repair for aberrant right subclavian artery aneurysm. Ann Thorac Cardiovasc Surg. 2014;20(Suppl):790-3.

41. Okwara CJ, Petrasek J, Gibson M, et al. Secondary aortoesophageal fistula associated with aneurysmal graft infection by Coxiella burnetii. ACG Case Rep J. 2016;3:169-71.

42. Tao M, Shlomovitz E, Darling G, et al. Secondary aorto-esophageal fistula after thoracic aortic aneurysm endovascular repair treated by covered esophageal stenting. World J Clin Cases. 2016;4:233-7.

43. Prokakis C, Koletsis E, Apostolakis E, et al. Aortoesophageal fistulas due to thoracic aorta aneurysm: surgical versus endovascular repair. Is there a role for combined aortic management? Med Sci Monit. 2008;14:Ra48-54.

44. Gimenez A, Franquet T, Erasmus JJ, et al. Thoracic complications of esophageal disorders. Radiographics 2002;22 Spec No:S247-58.

45. Hagspiel KD, Turba UC, Bozlar U, et al. Diagnosis of aortoenteric fistulas with CT angiography. J Vasc Interv Radiol. 2007; 18:497-504.

46. Christensen JD, Heyneman LE. Case of the season: aortoesophageal fistula complicating thoracic aortic aneurysm stent graft repair. Semin Roentgenol. 2009;44:4-7.

47. Hakim S, Gjeorgjievski M, Garg L, et al. Atypical aortoesophageal fistula with atypical and delayed presentation and negative imaging studies. Case Rep Gastrointest Med. 2016;2016:7219034.

48. Akaraviputh T, Sriprayoon T, Prachayakul V, et al. Endoscopic diagnosis of secondary aortoesophageal fistula. Endoscopy. 2008;40(Suppl 2):E90.

49. Isasti G, Gomez-Doblas JJ, Olalla E. Aortoesophageal fistula: an uncommon complication after stent-graft repair of an aortic thoracic aneurysm. Interact Cardiovasc Thorac Surg. 2009;9:683-4.

50. Yamanaka K, Nonaka M, Iwakura A, et al. Repair of aortoesophageal fistula after total aortic arch grafting. Interact Cardiovasc Thorac Surg. 2011;12:655-6.

51. Sosnowik D, Greenberg R, Bank S, et al. Aortoesophageal fistula: early and late endoscopic features. Am J Gastroenterol. 1988;83:1401-4.

52. Miller RG, Robie DK, Davis SL, et al. Survival after aberrant right subclavian artery-esophageal fistula: case report and literature review. J Vasc Surg. 1996;24:271-5.

53. Yuki M, Amano Y, Komazawa Y, et al. Unsedated transnasal small-caliber esophagogastroduodenoscopy in elderly and bedridden patients. World J Gastroenterol. 2009;15:5586-91. 
54. Bakhshandeh AR, Salehi M, Radmehr H, et al. A case of aortoesophageal fistula. Asian Cardiovasc Thorac Ann. 2011;19:419-21.

55. Ctercteko G, Mok CK. Aorta-esophageal fistula induced by a foreign body: the first recorded survival. J Thorac Cardiovasc Surg. 1980;80:233-5.

56. von Segesser LK, Tkebuchava T, Niederhauser U, et al. Aortobronchial and aortoesophageal fistulae as risk factors in surgery of descending thoracic aortic aneurysms. Eur J Cardiothorac Surg. 1997;12:195-201.

57. Coselli JS, Crawford ES. Primary aortoesophageal fistula from aortic aneurysm: successful surgical treatment by use of omental pedicle graft. J Vasc Surg. 1990;12:269-77.

58. Cheng L, Zhu J, Liu X, et al. A successful three-stage surgical treatment for aortoesophageal fistula after thoracic endovascular aortic repair and esophageal stent repair. Ann Thorac Surg. 2016;102:e503-5.

59. Afifi RO, Mushtaq HH, Sandhu HK, et al. Successful multistaged surgical management of secondary aortoesophageal fistula with graft infection. Ann Thorac Surg. 2016;101:e203-5.

60. Kieffer E, Chiche L, Gomes D. Aortoesophageal fistula: value of in situ aortic allograft replacement. Ann Surg. 2003;238:283-90.

61. Saito A, Motomura N, Hattori O, et al. Outcome of surgical repair of aorto-eosophageal fistulas with cryopreserved aortic allografts. Interact Cardiovasc Thorac Surg. 2012;14:532-7.

62. Iguchi A, Miyazaki S, Akimoto H, et al. Successful management of secondary aortoesophageal fistula with graft infection. Thorac Cardiovasc Surg. 2001;49:126-8.

63. McFaddin DM, Dang C. Management of aortoesophageal fistula. A case report. Am Surg. 1985;51:548-50.

64. Strug BS, Saltzman DA, Feldman MI, et al. Aorto-esophageal fistula. Cardiovasc Res Cent Bull. 1979;18:34-8.

65. Gomez-Alonso A, Lozano F, Cuadrado F, et al. Traumatic aortoesophageal fistula. J Thorac Cardiovasc Surg. 1984;87:148-9.

66. Reedy FM. Embolization of aortoesophageal fistula: a new therapeutic approach. J Vasc Surg. 1988;8:349-50.

67. Shiraishi S, Watarida S, Matsubayashi K, et al. Successful management of an aortoesophageal fistula resulting from an aneurysm of the thoracic aorta with a covered stent. J Cardiovasc Surg (Torino). 2002;43:95-8.

68. Okita Y, Yamanaka K, Okada K, et al. Strategies for the treatment of aorto-oesophageal fistula. Eur J Cardiothorac Surg. 2014;46:894-900.

69. Topel I, Stehr A, Steinbauer MG, et al. Surgical strategy in aortoesophageal fistulae: endovascular stentgrafts and in situ repair of the aorta with cryopreserved homografts. Ann Surg. 2007;246:853-9.
70. Grabenwoger M, Alfonso F, Bachet J, et al. Thoracic Endovascular Aortic Repair (TEVAR) for the treatment of aortic diseases: a position statement from the European Association for CardioThoracic Surgery (EACTS) and the European Society of Cardiology (ESC), in collaboration with the European Association of Percutaneous Cardiovascular Interventions (EAPCI). Eur Heart J. 2012;33:1558-63.

71. Gavens E, Zaidi Z, Al-Jundi W, et al. Aortoesophageal fistula after endovascular aortic aneurysm repair of a mycotic thoracic aneurysm. Int J Vasc Med. 2011;2011:649592.

72. Yavuz S, Kanko M, Ciftci E, et al. Aortoesophageal fistula secondary to thoracic endovascular aortic repair of a descending aortic aneurysm rupture. Heart Surg Forum. 2011;14:E249-51.

73. Czerny M, Eggebrecht $\mathrm{H}$, Sodeck G, et al. New insights regarding the incidence, presentation and treatment options of aorto-oesophageal fistulation after thoracic endovascular aortic repair: the European Registry of Endovascular Aortic Repair Complications. Eur J Cardiothorac Surg. 2014;45:452-7.

74. Chiesa R, Tshomba Y, Kahlberg A, et al. Management of thoracic endograft infection. $\mathrm{J}$ Cardiovasc Surg (Torino). 2010;51:15-31.

75. Metz R, Kimmings AN, Verhagen HJ, et al. Aortoesophageal fistula successfully treated by endovascular stent-graft. Ann Thorac Surg. 2006;82:1117-9.

76. Kasai K, Ushio A, Tamura Y, et al. Conservative treatment of an aortoesophagial fistula after endovascular stent grafting for a thoracic aortic aneurysm. Med Sci Monit. 2011;17:Cs39-42.

77. Numan F, Gulsen F, Cantasdemir M, et al. Percutaneous treatment of an infected aneurysmal sac secondary to aortoesophageal fistula with a history of stent-graft treatment for thoracic aortic aneurysm. Cardiovasc Intervent Radiol. 2012;35:690-4.

78. Burks JA Jr, Faries PL, Gravereaux EC, et al. Endovascular repair of bleeding aortoenteric fistulas: a 5-year experience. J Vasc Surg. 2001;34:1055-9.

79. Mosquera VX, Marini M, Pombo-Felipe F, et al. Predictors of outcome and different management of aortobronchial and aortoesophageal fistulas. J Thorac Cardiovasc Surg. 2014;148:3020-6.

80. Pohl J, Borgulya M, Lorenz D, et al. Endoscopic closure of postoperative esophageal leaks with a novel over-the-scope clip system. Endoscopy. 2010;42:757-9.

81. Matsuoka K, Ito A, Murata Y, et al. Severe mediastinitis and pericarditis after transbronchial needle aspiration. Ann Thorac Surg. 2015;100:1881-3.

82. Mudumbi S, Velazquez-Avina J, Neumann H, et al. Anchoring of self-expanding metal stents using the over-the-scope clip, and a technique for subsequent removal. Endoscopy. 2014;46:1106-9. 